\title{
OPEN Postural adjustments impairments in elderly people with chronic low back pain
}

\author{
Daniela Rosa Garcez ${ }^{1,2}$, Gizele Cristina da Silva Almeida ${ }^{3,4}$, Carlos Felipe Oliveira Silva ${ }^{3}$, \\ Tainá de Souza Nascimento ${ }^{3}$, Anselmo de Athayde Costa e Silva ${ }^{5}$, \\ Ana Francisca Rozin Kleiner ${ }^{6}$, Givago da Silva Souza ${ }^{4,2}$, Elizabeth Sumi Yamada ${ }^{2,7}$ \& \\ Bianca Callegari ${ }^{3,5,8 \bowtie}$
}

Chronic low back pain (CLBP) is associated with postural control impairments and is highly prevalent in elderly people. The objective of this study is to verify whether anticipatory postural adjustments (APAs) and compensatory postural adjustments (CPAs) are affected by CLBP in elderly people by assessing their postural control during a self-initiated perturbation paradigm induced by rapid upper arm movement when pointing to a target. The participants' lower limb muscle onset and center of pressure (COP) displacements were assessed prior to perturbation and throughout the entire movement. $T_{0}$ moment (i.e., the beginning of the movement) was defined as the anterior deltoid (DEL) onset, and all parameters were calculated with respect to it. The rectus femoris (RT), semitendinosus (ST), and soleous (SOL) showed delayed onset in the CLBP group compared with the control group: RF (control: $-0.094 \pm 0.017 \mathrm{~s}$; CLBP: $-0.026 \pm 0.012 \mathrm{~s}, \mathrm{t}=12, \mathrm{p}<0.0001$ ); ST (control: $-0.093 \pm 0.013 \mathrm{~s}$; CLBP: $-0.018 \pm 0.019 \mathrm{~s}, \mathrm{t}=12, \mathrm{p}<0.0001$ ); and SOL (control: $-0.086 \pm 0.018 \mathrm{~s}$; CLBP: $-0.029 \pm 0.015 \mathrm{~s}$, $t=8.98, p<0.0001$ ). In addition, COP displacement was delayed in the CLBP group (control: $-0.035 \pm 0.021 s ; C L B P:-0.015 \pm 0.009 s, t=3 ; p=0.003$ ) and presented a smaller amplitude during APA $\mathrm{COP}_{\text {APA }}$ [control: $0.444 \mathrm{~cm}(0.187 ; 0.648) ;$ CLBP: $0.228 \mathrm{~cm}(0.096 ; 0.310), \mathrm{U}=53, \mathrm{p}=0.012$ ]. The CLBP group required a longer time to reach the maximum displacement after the perturbation (control: $0.211 \pm 0.047 \mathrm{~s} ; \mathrm{CLBP} 0.296 \pm 0.078 \mathrm{~s}, \mathrm{t}=3.582, \mathrm{p}=0.0013$ ). This indicates that CLBP elderly patients have impairments to recover their postural control and less efficient anticipatory adjustments. Our results suggest that people with CLBP have altered feedforward hip and ankle muscle control, as shown from the SOL, ST, and RT muscle onset. This study is the first study in the field of aging that investigates the postural adjustments of an elderly population with CLBP. Clinical assessment of this population should consider postural stability as part of a rehabilitation program.

Low back pain (LBP) is one of the most frequent symptoms reported by older people. It is defined as any pain or discomfort between one's last ribs and the lower gluteal line, with or without irradiation symptoms to the lower limbs ${ }^{1}$. Pain duration is one of the criteria for classifying LBP types. Long-duration pain is defined as chronic low back pain $(\mathrm{CLBP})^{2}$. The most typical classification of CLBP is nonspecific CLBP, which refers to cases in which the etiology of a patient's pain is unidentifiable ${ }^{3}$. In addition, CLBP is a risk factor for incapacity and invalidity ${ }^{3,4}$; it is the leading cause of functional limitations associated with the performance of daily living activities ${ }^{3,4}$, with a higher prevalence in women aged 60-69 years ${ }^{5}$. In fact, aging and CLBP are important factors that affect an individual's postural control ${ }^{6-8}$.

\footnotetext{
${ }^{1}$ University Hospital Bettina Ferro de Souza, Federal University of Pará, R. Augusto Corrêa, n1, Belém, Pará 66075-110, Brazil. ${ }^{2}$ Neuroscience and Cell Biology Graduate Program, Federal University of Pará, R. Augusto Corrêa, n 1, Belém, Pará 66075-110, Brazil. 'Laboratory of Human Motricity Sciences, Federal University of Pará, Av. Generalíssimo Deodoro 01, Belém, Pará 66050-160, Brazil. ${ }^{4}$ Tropical Medicine Center, Federal University of Pará, Av. Generalíssimo Deodoro 92, Belém, Pará 66050-240, Brazil. ${ }^{5}$ Master's Program in Human Movement Sciences, Federal University of Pará, Av. Generalíssimo Deodoro 01, Belém, Pará 66050-160, Brazil. ${ }^{6}$ Department of Physiotherapy, Federal University of São Carlos, Rodovia Washington Luiz km235, caixa postal 676, São Carlos, São Paulo 13565-905, Brazil. ${ }^{7}$ Graduate Program in Medical Sciences and Oncology, Federal University of Pará, Rua dos Mundurucus 4487, Belém, Pará 66073-005, Brazil. ${ }^{8}$ Instituto de Ciências da Saúde, Avenida Generalíssimo Deodoro, n¹, Belém, Pará 66055-240, Brazil. ${ }^{\circledR}$ email: callegaribi@uol.com.br
} 
Postural control is necessary to maintain one's center of mass within the basis of support, preventing falls. When perceiving an upcoming perturbation that may result in center of mass (COM) displacement, our central nervous system uses anticipatory postural adjustments (APAs) and compensatory postural adjustments $(\mathrm{CPAs})^{9,10}$. Hence, when individuals execute voluntary movements that generate self-initiated perturbations, APAs are triggered before their movements begin to prevent or minimize any effects from such perturbation ${ }^{10,11}$. This is observed during rapid arm movement, such as in a pointing task at maximum velocity. APAs are represented by postural muscle activations beginning from -150 to $-100 \mathrm{~ms}$ prior to the focal movement, in a feedforward centrally programmed mechanism ${ }^{12}$. CPAs are a feedback-based control mechanism that restore balance through muscle activation following perturbation ${ }^{7,10}$. Center of pressure (COP) displacements are classically described to access APAs (measured as the onset of COP displacement and its amplitude prior to perturbation) and CPAs (described as the recovery time necessary to set back to the initial position, and the COP amplitude after perturbation $)^{7,8,13,14}$.

Studies have reported an important relation between APAs and CPAs, indicating that the greater the anticipatory adjustments, the less necessary are the compensatory adjustments to maintain one's stability ${ }^{7,10}$.

Aging affects postural control, increasing both the risk of falls and the fear of falling ${ }^{15}$. Previous studies have reported that the elderly population presented the delayed onset of postural muscles during APAs ${ }^{7,14,16}$, decreased APAs and/or increased CPAs postural muscle activation ${ }^{7}$, different muscle patterns or strategies to maintain posture $^{8,14,17}$, and delayed COP onset during APAs, when compared with young people ${ }^{8,14}$.

Postural control in people with CLBP has been described in the literature, albeit with inconclusive evidence. Most studies are primarily focused on the trunk musculature and young population, using self-initiated perturbation in the upright posture ${ }^{6,18}$. Differences in spinal erector muscle were investigated between healthy and CLBP adults. Some studies indicated a delay in the onset of this muscle ${ }^{18,19}$ in CLBP individuals, whereas others showed no differences between groups ${ }^{20}$. A recent systematic review reported that the onset of abdominal muscles during APAs was delayed in the CLBP population when compared with healthy controls ${ }^{6}$. However, the authors stated that evidence other than muscle latency, such as those based on measures from force platforms or kinematics, are not available in the literature. Once changes caused by CLBP in APAs onsets affect the movements and forces to which one's trunk is exposed during an activity, its functional consequences remain unclear ${ }^{21}$. In addition, only a few APA studies in individuals with CLBP included investigations pertaining to lower limb muscles ${ }^{22,23}$.

Hence, the literature provides limited evidence regarding changes in postural control in CLBP patients. Moreover, such studies excluded the elderly population and, therefore, do not provide information regarding postural control in older people with LBP. Evaluating postural strategies and balance is paramount when assessing and rehabilitating elderly patients, since they pose a high risk of falling, particularly when performing daily life activities.

This study aims to investigate the differences in APAs and CPAs in elderly people with and without CLBP by assessing their postural control after a self-initiated perturbation paradigm. We hypothesize that elderly people with CLBP will present delayed and increased COP displacements during the APA phase owing to a higher COP displacement during the CPA period. This implies that they will require more time to restore postural control. Additionally, we hypothesize that muscle onset during APAs will be delayed in elderly people with CLBP.

\section{Materials and methods}

The cross-sectional observational study performed in this investigation was approved by the Ethics Committee of the Federal University of Pará (protocol \#25317119.4.0000.0018) as well as the Observational Studies in Epidemiology (STROBE) Statement. Written informed consent was obtained from all participants before the study was started. The study was performed from March 2019 to March 2020.

Participants. Thirty elderly participants participated in the present study. They were segregated into two groups, matched by age, height, and weight: $\operatorname{CLBP}(\mathrm{n}=15 ; 4$ males; 11 females $)$ and control $(\mathrm{n}=15 ; 3$ males; 12 females). The elderly with CLBP were submitted to a geriatrician-confirmed diagnosis of nonspecific CLBP, of which the inclusion criteria were as follows: (1) history of chronic unilateral or bilateral CLBP ( $\geq 3$ months) without pain referral to their lower limbs; (2) the ability to stand and walk independently; (3) having a score $\leq 2$ on the numeric pain rating scale (NRS) $)^{13,24,25}$; and (4) the ability to understand verbal commands for executing the required tasks ${ }^{26,27}$. The exclusion criteria were nonmechanical CLBP (e.g., fracture, malignancy, and infection); radicular signs; history of back surgery; diagnosis of inflammatory joint disease; severe osteoporosis; metabolic or neuromuscular diseases; other chronic pain pathologies; any major circulatory, respiratory, neurological, or cardiac diseases; or cognitive deficit.

For the control group, the inclusion criteria were not having CLBP throughout the previous year or back pain lasting longer than one week in the previous 3 years. The exclusion criteria were previous histories of neurological or musculoskeletal disorders that induced visible gait abnormalities. To better characterize the sample, both groups completed the Oswestry disability index (ODI) $)^{28}$ for assessing function disability. Table 1 summarizes the main characteristics of the participants from both groups.

Experimental setup for APA assessment. The participants stood with bare feet on a force platform and were instructed to use a self-selected pleasant position such that the mid-point of their heels were separated by a distance equal to the width of their shoulder, with feet externally rotated up to $15^{\circ}$, which is considered a natural comfortable position. They were instructed to observe a horizontal bar that was placed in front of them, $2 \mathrm{~m}$ above the floor, and $2 \mathrm{~m}$ away from the participants' feet, with a light-emitting diode (LED) aligned to their right shoulder. The participants stood with their arms relaxed down along their bodies, and their right index finger pointing to the ground. Subsequently, they were asked to move the arm, using their right index fingers to point 


\begin{tabular}{|l|l|l|l|}
\hline & CTL group & CLBP group & p-value \\
\hline Age (years) & $70.2 \pm 4.6$ & $70.13 \pm 6.5$ & 0.9745 \\
\hline Height $(\mathrm{m})$ & $1.56 \pm 0.04$ & $1.55 \pm 0.03$ & 0.7935 \\
\hline Weight $(\mathrm{kg})$ & $54.07 \pm 2.21$ & $54.53 \pm 2.19$ & 0.5676 \\
\hline NRS & - & $1.53 \pm 0.63$ & - \\
\hline ODI $(\%) \#$ & $0(0 ; 4)$ & $12.5(10 ; 16)$ & $<0.0001$ \\
\hline
\end{tabular}

Table 1. Sample characteristics. Data expressed as mean \pm SD if presented with a normal distribution, or median and percentiles if presented with nonparametric distribution. The level of significance was 0.05 . NRS numeric pain rating scale, ODI Oswestry disability index.

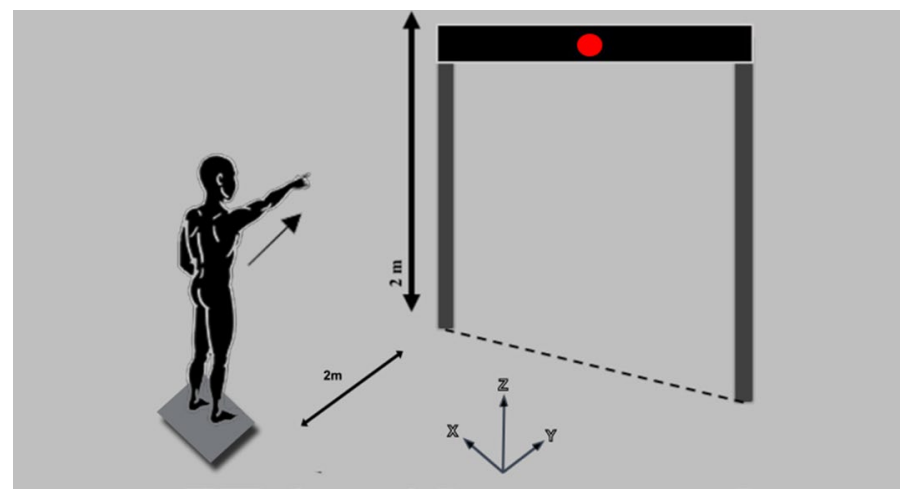

Figure 1. Experimental setup for task showing a participant in final posture. Central diode of the bar was placed exactly in front of participants' right shoulder. Participants were asked to point their index finger, with extended elbow, at the central diode whenever it was turned on.

the LED every time it was turned on in a ten-trial round. Electrical activity in the anterior deltoid (DEL) was visually verified prior to switching on the LED to ensure that the deltoid muscle was relaxed. For every trial, the participants were instructed to extend their elbows and raise their arms as fast as possible after perceiving the visual stimuli, maintaining their arm in the air for a few seconds and then moving it back to the initial position (Fig. 1).

Kinetic, kinematic, and electromyographic recording. In this study, a force platform (Biomec 400041, EMG System) sampled at $100 \mathrm{~Hz}$ was used to record three-dimensional ground reaction forces. Using the obtained data, we computed the coordinates of the participants' COP in the anteroposterior direction. A threedimensional motion analysis system (Simi Motion, Simi, Germany) with three cameras at a sampling frequency of $120 \mathrm{~Hz}$ was used to record the participants' movements. Each of them comprised four infrared reflective markers placed at the main joints of their right upper limb (i.e., index, wrist, elbow, and shoulder). Surface electromyographic (EMG) data were recorded from the participants' dominant-side leg muscles: tibialis anterior (TA), soleus (SOL), rectus femoris (RF), semitendinosus (ST), and anterior deltoid (DEL), using an EMG device (Emgsys 30,306, EMG System do Brasil, Brazil), with a sampling rate of $2 \mathrm{kHz}$ and a frequency spectrum of 20-500 Hz. The EMG signals were amplified (4000) and digitized with a 16-bit resolution. The participants' skin was prepared for the placement of $\mathrm{Ag} / \mathrm{AgCl}$ electrodes (Medtrace 200-Kendall, Canada) using Nuprep (Weaver and Company, Aurora, United States) and an alcohol-based sanitizer. By following the recommendations of the Surface Electromyography for Non-Invasive Assessment of Muscles guidelines ${ }^{29}$, we placed active electrodes on their muscles at $20 \mathrm{~mm}$ intervals and the reference electrode on their right fibular malleolus.

Data analysis. Raw EMG signals were bandpass filtered between 20 and $400 \mathrm{~Hz}$, full-wave rectified, and bidirectionally filtered using a $6 \mathrm{~Hz}$ low-pass, second-order, zero-lag Butterworth filter. Muscle onset (concerning both the activation/inhibition of a muscle) was detected in relation to $\mathrm{T}_{0}$ via visual inspection performed by two blinded examiners. The low-pass filtering generated a smooth envelope, which, in combination with the raw signal, was used for the visual identification of the muscle onsets. $\mathrm{T}_{0}$ moment (i.e., the beginning of the movement) was defined as the onset of DEL. After the onset of each trial, we calculated the timing of each muscle activation with reference to the DEL onset ${ }^{16,18}$.

Triggers of kinematics and force platform data were provided to two channels of the EMG to permit data synchronization and offline analysis using MatLab programs (MathWorks, Natick, MA, USA). Ten trials were performed for the calculations. The kinematic parameters of the participants' arm movements extracted by the index finger (trajectory and tangential velocity profile) were analyzed. The raw coordinate data on the $\mathrm{x}^{-}, \mathrm{y}^{-}$, and 


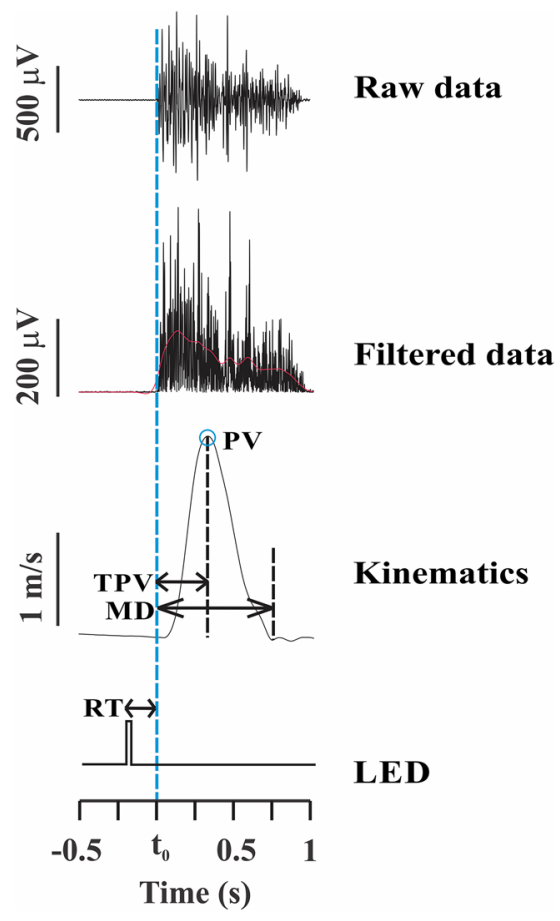

Figure 2. DEL onset detection after LED stimuli and using kinematic parameters. Dashed blue line represents $\mathrm{T}_{0}$ moment of the task.

$\mathrm{z}$-axes were generated from the video analysis and then filtered using a bidirectional $10-\mathrm{Hz}$ low-pass, secondorder Butterworth filter. The kinematic variables were (1) the reaction time (RT), measured as the time interval from the LED stimuli to $\mathrm{T}_{0}$ moment; (2) the peak velocity (PV): the maximum velocity reached by a participant's arm during the pointing task; (3) the time to peak velocity (TPV), measured as the time from the $\mathrm{T}_{0}$ moment to the maximum peak velocity moment; (4) the total movement duration (MD): the time interval between the $T_{0}$ moment and the end of the trial when a participant's index finger stops pointing to the diode (velocity returns to zero); and (5) the index tangential velocity profile, which was calculated by the ratio of acceleration time (i.e., the fraction of movement time required to reach peak velocity) to the total movement duration (ACC/MD). This velocity profile is classically described as reflecting the content of motor planning ${ }^{30}$. More specifically, asymmetries in the relative acceleration duration (i.e., ACC/MD) demonstrate different motor plans for the execution and control of arm movements.

Figure 2 presents the DEL onset detection after the LED stimuli and the kinematic parameters.

COP displacements in the anteroposterior direction were calculated based on previous studies ${ }^{31}$. The baseline activity used for the calculation was from -500 to $-400 \mathrm{~ms}$ in relation to the $\mathrm{T}_{0}$ moment. The displacement in the anteroposterior COP dimension was analyzed and four variables were derived from it. The first two variables were anticipatory in nature, whereas the other two variables were compensatory in nature: (1) the beginning of the COP displacement before $\mathrm{T}_{0}$ moment, measured as the time when the COP displacement was smaller than the mean of its baseline value plus 2 standard deviation $(\mathrm{SD})\left(\mathrm{COP}_{\text {onset }}\right)(\text { Fig. } 3 \mathrm{~B})^{13} ;(2)$ the anteroposterior COP displacement at $\mathrm{T}_{0}$ moment (measured from the baseline amplitude), which is anticipatory in nature, known as the amplitude of the COP displacement at $\mathrm{T}_{0}\left(\mathrm{COP}_{\mathrm{APA}}\right)(\mathrm{Fig} .3 \mathrm{~A})$; (3) the peak displacement measured, which is compensatory in nature $\left(\mathrm{COP}_{\text {disp }}\right)$, as the maximum displacement after $\mathrm{T}_{0}$ moment (Fig. $\left.3 \mathrm{~A}\right)$; and (4) the time to reach this peak maximum displacement $\left(\mathrm{COP}_{\text {timetopeak }}\right)(\text { Fig. } 3 \mathrm{~B})^{7,8,14}$.

Statistical analysis. GraphPad Prism 6 (San Diego, California, USA) and BioEstat 5.3 (Belém, Pará, Brazil) for Windows was used to perform the statistical procedures of this study. The data distribution was tested via the Shapiro-Wilk test. Medians with first and third quartiles were reported for nonparametric outcomes, whereas the mean and SD for parametric outcomes. Data were compared between groups. Unpaired t-test was performed to compare the parametric outcomes (age, height, weight, muscles onset during APAs, $\mathrm{COP}_{\text {onset }}$, and $\mathrm{COP}_{\text {timetopeak; }}$ and the kinematic parameters PV, MD, RT, and ACC/MD), whereas the Mann-Whitney test was performed to compare nonparametric outcomes (ODI score, time to peak velocity, $\mathrm{CO}_{\mathrm{APA}}$, and $\mathrm{COP}_{\text {disp) }}$. For all these statistical treatments, the significance level was $\mathrm{p} \leq 0.05$.

\section{Results}

Kinematic characteristics. Table 2 summarizes the pointing task kinematic parameters. In this study, no statistical differences were observed between groups in terms of kinematic features. 

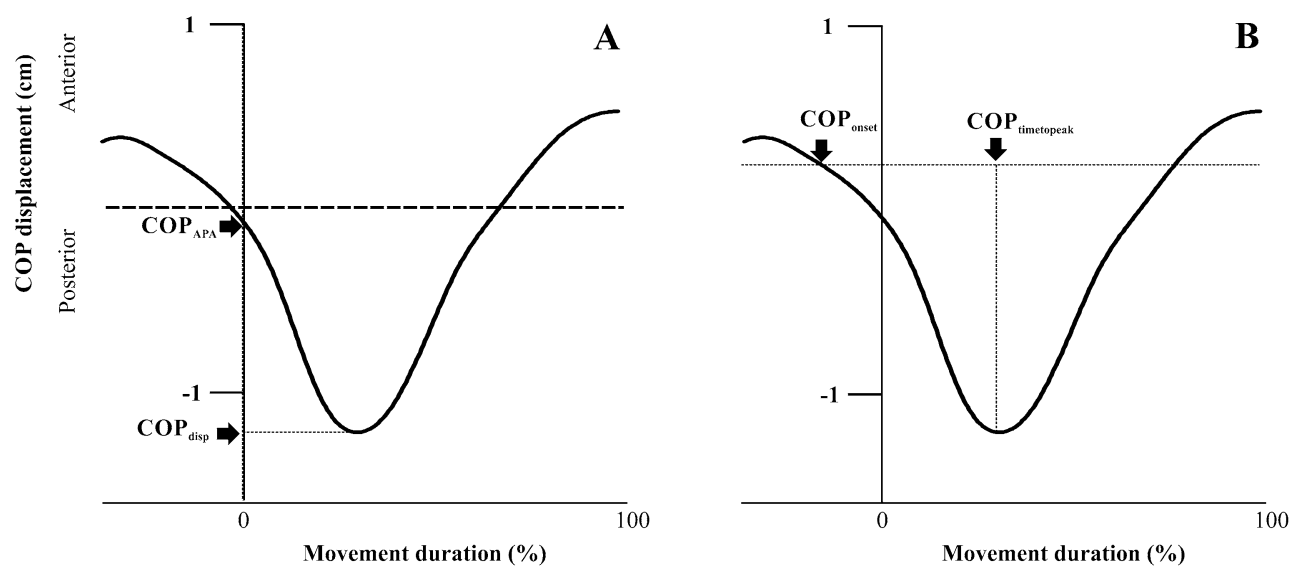

Figure 3. (A, B) Anteroposterior COP displacement (y-axis) when a participant moves his/her arm. Dashed line represents movement onset. Four variables: (1) $\mathrm{COP}_{\mathrm{APA}}$, amplitude of backward COP displacement at $\mathrm{T}_{0}$; (2) COPdisp, maximum backward displacement after $\mathrm{T}_{0 ;}(\mathbf{A})$; (3) COPonset, time of initial backward displacement before $\mathrm{T}_{0}$; and (4) COPtimetopeak, time to reach maximum displacement (B).

\begin{tabular}{|l|l|l|l|}
\hline & Control group & CLBP group & p-value \\
\hline Reaction time (s) & $0.160 \pm 0.031$ & $0.177 \pm 0.068$ & 0.407 \\
\hline Peak velocity (m/s) & $5.375 \pm 1.44$ & $4.709 \pm 1.062$ & 0.161 \\
\hline Acceleration time/movement duration & $0.462 \pm 0.095$ & $0.460 \pm 0.060$ & 0.959 \\
\hline Time to peak velocity (s) & $0.348(0.295 ; 0.377)$ & $0.315(0.290 ; 0.353)$ & 0.290 \\
\hline Movement duration (s) & $0.669 \pm 0.080$ & $0.676 \pm 0.092$ & 0.815 \\
\hline
\end{tabular}

Table 2. Comparison between kinematic parameters. Data expressed as mean $\pm S D$ if presented with a normal distribution, or median and percentiles if presented with nonparametric distribution.

Muscle latency. Figure 4 presents each muscle onset of a single trial of one control elderly participant and one CLBP elderly participant.

Proximal muscles (RF and ST) and the distal muscle (SOL) showed delayed onset in the CLBP group when compared with the control group (Fig. 5). The onset of APA activity was as follows: RF (control: $-0.094 \pm 0.017 \mathrm{~s}$; CLBP: $-0.026 \pm 0.012 \mathrm{~s}, \mathrm{t}=12, \mathrm{p}<0.0001$ ); ST (control: $-0.093 \pm 0.013 \mathrm{~s}$; CLBP: $-0.018 \pm 0.019 \mathrm{~s}, \mathrm{t}=12$, $\mathrm{p}<0.0001$ ); TA (control: $-0.035 \pm 0.009 \mathrm{~s}$; CLBP: $-0.025 \pm 0.017 \mathrm{~s}, \mathrm{t}=1.96, \mathrm{p}=0.059$ ); and SOL (control: $-0.086 \pm 0.018 \mathrm{~s} ;$ CLBP: $-0.029 \pm 0.015 \mathrm{~s}, \mathrm{t}=8.98, \mathrm{p}<0.0001)$.

Displacements of COP. Figure 6 presents the anteroposterior COP displacement of a single trial of one control elderly participant and one CLBP elderly participant.

Figure 7 depicts the $\mathrm{COP}_{\text {onset }}(\mathrm{A}), \mathrm{COP}_{\text {timetopeak }}(\mathrm{B}), \mathrm{COP}_{\mathrm{APA}}(\mathrm{C})$, and $\mathrm{COP}_{\text {disp }}(\mathrm{D})$. The CLBP group showed delayed $\mathrm{COP}_{\text {onset }}$ (control: $-0.035 \pm 0.021 \mathrm{~s} ; \mathrm{CLBP}:-0.015 \pm 0.009, \mathrm{t}=3 ; \mathrm{p}=0.003$ ) and a smaller $\mathrm{COP}_{\mathrm{APA}}$ (control: $0.444 \mathrm{~cm}(0.187 ; 0.648)$; CLBP: $0.228 \mathrm{~cm}(0.096 ; 0.310, \mathrm{U}=53, \mathrm{p}=0.012)$ compared with the control group (Fig. 7A,C). Although both groups reached a similar $\mathrm{COP}_{\text {disp }}$ after perturbation [control: $0.849 \mathrm{~cm}(0.703 ; 1.418)$; CLBP: $1.013 \mathrm{~cm}(0.666 ; 1.162), \mathrm{U}=105, p=0.766)]$, the CLBP group required more time to reach it, presenting a higher $\mathrm{COP}_{\text {timetopeak }}$ compared with the control group (control: $0.211 \pm 0.047$, CLBP $0.296 \pm 0.078 \mathrm{~s}, \mathrm{t}=3.582$, $\mathrm{p}=0.0013)($ Fig. 7B,D).

\section{Discussion}

The study was conducted to investigate the effects of CLBP on APAs and CPAs in elderly people during a selfinitiated perturbation paradigm. In general, our results demonstrated a delayed activation of the lower limb proximal muscles (RF and ST) and the distal muscle (SOL) in elderly patients with CLBP compared with the match-aged control elderly. During the APA phase, the COP displacement delayed and presented a smaller amplitude in the CLBP elderly. No differences was observed in the peak of COP displacement during the CPA period. However, the CLBP elderly required more time to reach it. Since a longer time for stabilizing posture is associated with poor postural control ${ }^{32}$ and impaired ability to recover postural stability throughout the movement (from the beginning through the end of one's arm movement $)^{13}$, the CLBP elderly patients have exhibited less efficient anticipatory adjustments and greater difficulty in recovering postural control during the compensatory phase. 

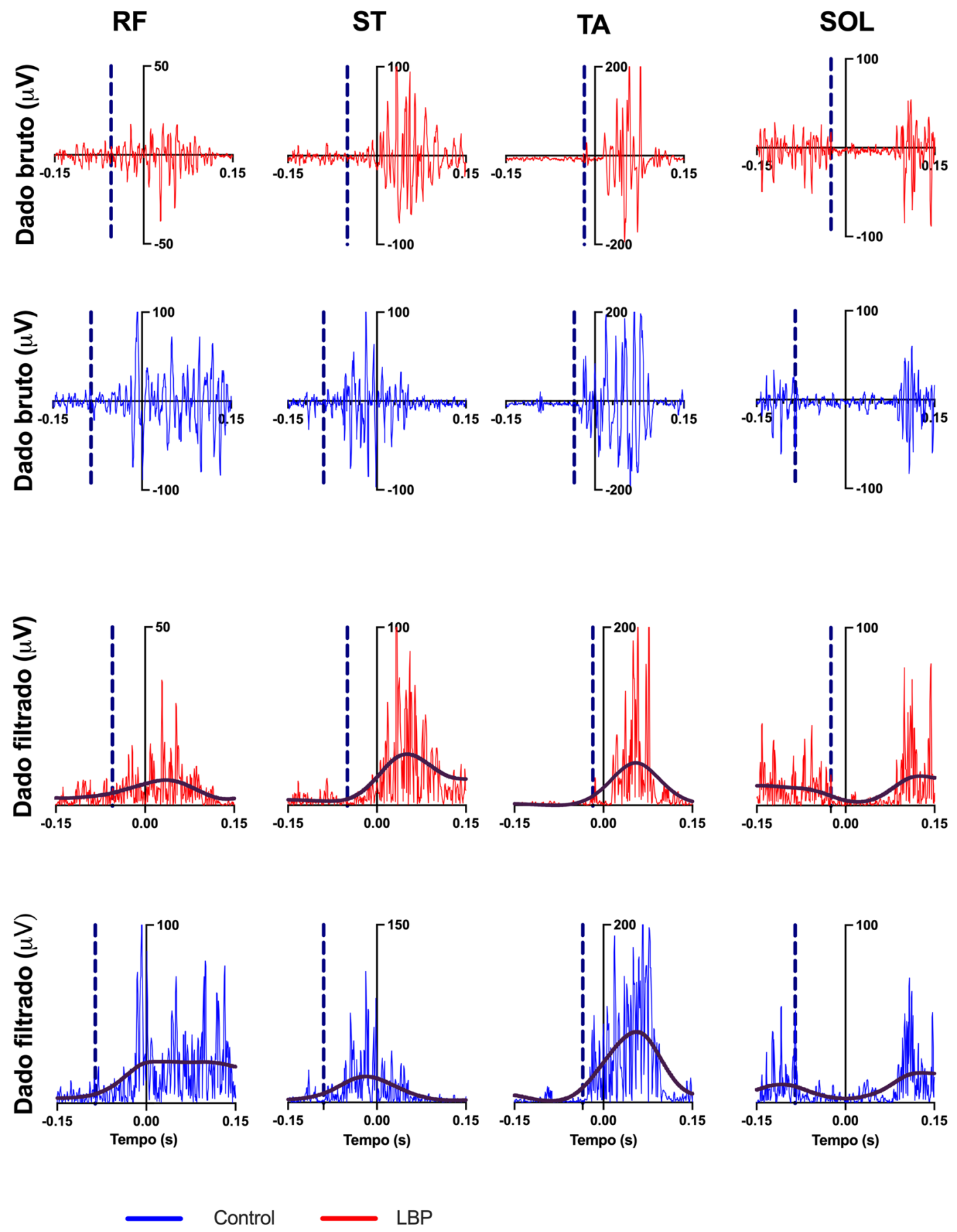

Figure 4. Raw and rectified $6 \mathrm{~Hz}$ low-pass filtered muscles activity of a typical participant of each group, recorded during one single trial. Vertical blue dashed line indicates muscles onset $\left(\mathrm{t}_{0}\right)$. Muscle abbreviations: $S T$ semitendinosus, $R F$ rectus femoris, $S O L$ soleous, $T A$ tibialis anterior. Control participants' anticipation compared with CLBP results.

Kinematic characteristics. Both groups presented similar kinematic features, demonstrating that they fully performed the task similarly, i.e., with the same perturbation magnitude. Studies regarding the pointing task paradigm demonstrated that a person's postural adjustments depended on velocity: the higher the speed, the worse was the postural adjustments ${ }^{8,17}$. The glenohumeral joint was subjected to opposing forces when the direction of shoulder motion was changed; acceleration and deceleration were involved when performing movement tasks. Accelerations caused by rotation forces in this joint resulted in multipoint reaction forces that may perturb other parts of an individual's body ${ }^{33}$. Hence, kinematic characteristics, such as limb acceleration peak ${ }^{13}$, mean speed ${ }^{17}$, or velocity peak ${ }^{8}$ of one's upper limb are typically used to guarantee similar perturbation mag- 


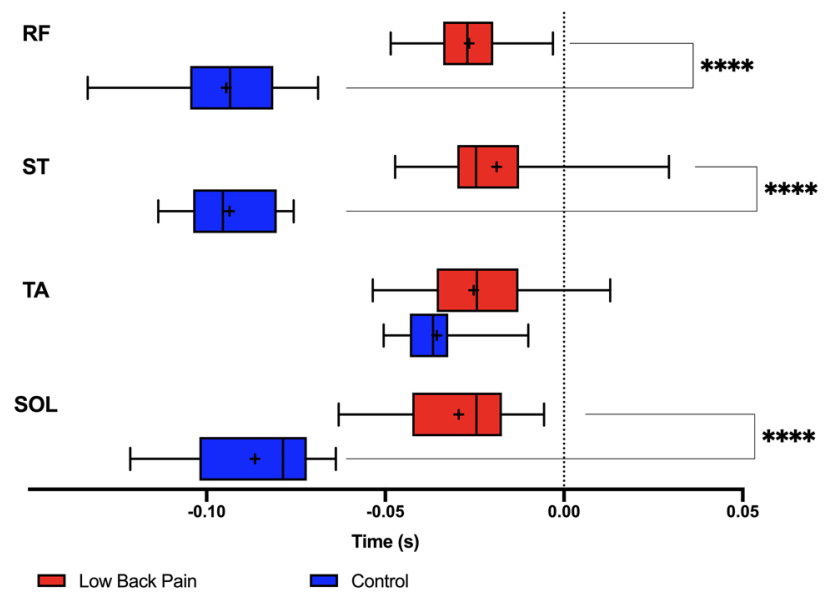

Figure 5. Muscle activity onsets for both control elderly and CLBP elderly participants. Muscle abbreviations: $R F$ rectus femoris, $S T$ semitendinosus, $S O L$ soleus, $T A$ tibialis anterior. Differences in latencies were significant when $\mathrm{p}<0.05\left(^{*}\right)$. Data expressed by central line $=$ median, box $=25$ and 75 percentiles, and whiskers $=\min$ and max values (mean values inside box marked as $\mathrm{X}$ ).

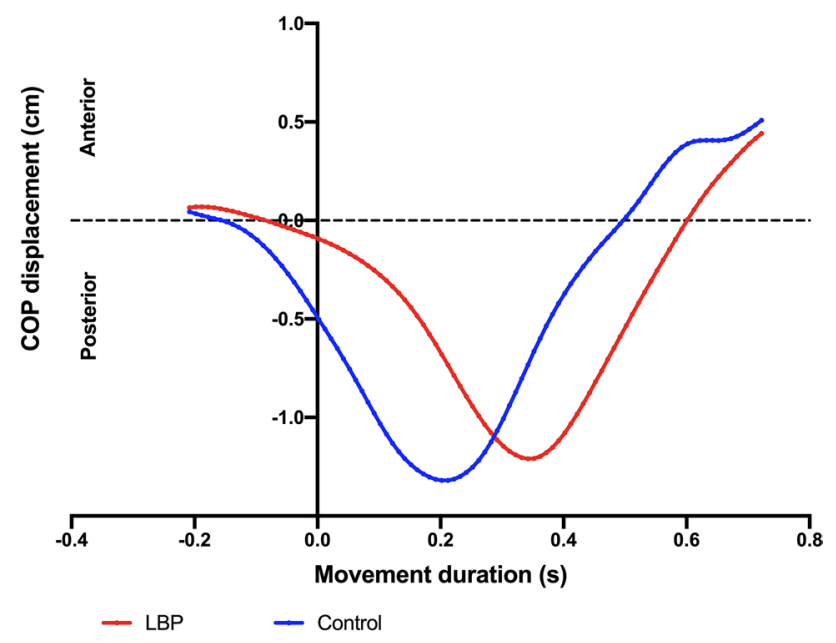

Figure 6. Anteroposterior COP displacement during arm movement of a typical participant recorded during one single trial. CLBP participants presented an earlier $\mathrm{COP}_{\text {onset }}$, a higher amplitude in $\mathrm{COP}_{\mathrm{APA}}$, and less time to reach the maximum displacement after $\mathrm{T}_{0}$ (lower $\mathrm{COP}_{\text {timetopeak }}$ ). No differences was observed between groups in $\mathrm{COP}_{\text {disp. }}$

nitudes. Furthermore, our results indicate that differences in postural adjustments between groups may have CLBP as a determining factor, rather than differences in the movement performed.

Altered muscular patterns in CLBP elderly. The results of the ST, RF, and SOL muscle onsets were consistent with our hypothesis, since the CLBP elderly patients presented delayed activation when compared with the control elderly participants. Some studies have previously reported delayed muscle activation related to CLBP during self-initiated perturbation in the upright posture; however, most of them primarily focused on young participants' trunk muscles ${ }^{6,18,19}$. A recent meta-analysis revealed a significant and substantial heterogeneity in muscle onsets ${ }^{6}$, stating that the onset of anterior trunk muscles (i.e., transverse abdominis, internal oblique, external oblique, and rectus abdominis) was delayed in CLBP participants when compared with healthy controls, with an acceptable amount of heterogeneity. Meanwhile, studies concerning posterior trunk muscles (i.e., spinal erectors) were controversial. Some outcomes indicated delayed muscle onset ${ }^{18}$ in CLBP individuals, whereas others showed no difference between groups ${ }^{18,20,24,34}$. Studies regarding lower limb muscles are scarce, thereby limiting the comparison of their results with ours. To our knowledge, only three related studies have been performed, among which one included the TA, RF, and $\mathrm{GAS}^{35}$, whereas the others included the $\mathrm{ST}^{23}$ and no GAS ${ }^{25}$. Sadeghi et al. ${ }^{25}$ discovered delayed activation of the trunk muscles in patients with CLBP; earlier activation of GAS was present in this group, and the authors speculated that CLBP patients adopted the ankle 
A

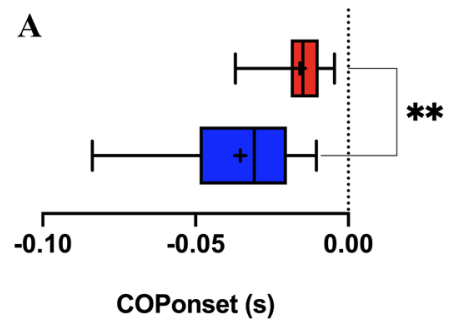

B

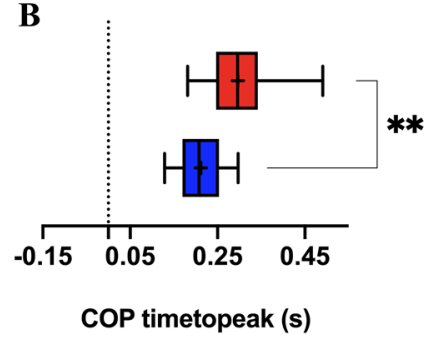

C

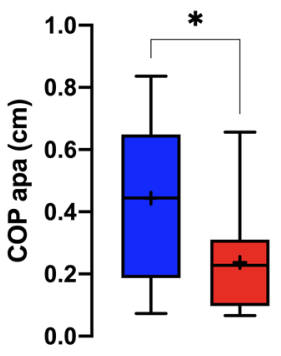

D

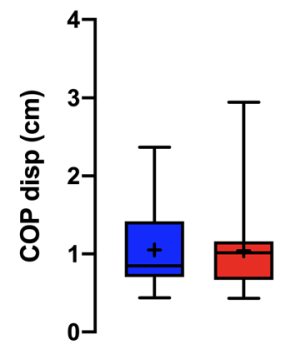

Low Back Pain

Control

Figure 7. (A) $\mathrm{COP}_{\text {onset; }}$ (B) $\mathrm{COP}_{\text {timetopeak; }}(\mathbf{C}) \mathrm{COP}_{\mathrm{APA}}$, and (D) $\mathrm{COP}_{\text {disp. }}$. Differences were significant when $p<0.05\left(^{*}\right)$. Data expressed by central line $=$ median, box $=25$ and 75 percentiles, and whiskers $=\min$ and $\max$ values (mean values inside box marked as $\mathrm{X}$ ).

strategy ${ }^{22}$ more frequently toward postural disturbance than healthy participants. Because that study included no other lower limb muscles, it was difficult to extend its results to ours.

Delayed muscle onset is also described in the literature as a dysfunctional joint strategy ${ }^{35}$ : delayed TA muscle activation in patients with nonspecific CLBP may occur when they perform challenging tasks, suggesting a dysfunctional ankle strategy in this population. Our results support this premise, since, in this study, the CLBP participants presented delayed SOL activation compared with the control participants.

Only one study ${ }^{22}$ investigated the differences between people with CLBP and healthy individuals with ST. Even though the results showed that the ST was delayed in CLBP participants, we cannot compare them with our ST findings because the authors did not investigate postural perturbation (i.e., the participants were performing a hip extension in prone).

In summary, our findings suggest that CLBP participants have altered feedforward motor control in the hip and ankle muscles, as demonstrated by delayed onsets of the ST, RF, and SOL. These findings are consistent with previously described strategies for young people with CLBP, such as decreased hip strategy ${ }^{36}$, increased trunk co-contraction activity ${ }^{37}$, and altered activation time of axial postural muscles ${ }^{6,18,24,34}$.

Impaired COP control in CLBP elderly. Our COP results partially confirmed our hypothesis, as we observed delayed $\mathrm{COP}_{\text {onset }}$ and reduced $\mathrm{COP}_{\mathrm{APA}}$ in CLBP elderly patients when compared with the control group. By analyzing the participants before performing the arm-moving task, we observed a backward displacement of COP in both groups. Rapid bilateral or unilateral upper limb flexion generated self-perturbation, in which a forward center of mass (COM) displacement resulted in a backward displacement of the $\mathrm{COP}^{38}$. This occurred prior to perturbation and continued throughout the compensatory phase until postural control was recovered, as indicated in our results ${ }^{8,39}$.

Previous studies used $\mathrm{COP}_{\text {onset }}$ and $\mathrm{COP}_{\mathrm{APA}}$ to assess the quality of APAs; it was reported that the smaller the onset and amplitude of the COP, the less efficient was the preparation for the expected perturbation ${ }^{7,8}$. Therefore, as hypothesized, the control group delivered more efficient APAs when compared with the CLBP elderly patients.

Contrary to the expected results, no differences was observed between groups in terms of the $\mathrm{COP}_{\text {disp }}$ after disturbance. However, the time required to reach it was higher in the CLBP elderly participants. The maximum displacement of COP after perturbation and the time to reach this peak guided the interpretations of compensatory adjustments after postural disturbance ${ }^{7,8,13,40}$. In general, it has been reported that smaller COP excursions after perturbation characterize better postural recovery ${ }^{7}$. We anticipated this result from the control group instead of the CLPB group; however, the phenomenon did not occur. Some authors have demonstrated a similar or even lower maximum COP displacement in adults with CLBP when compared with healthy participants ${ }^{13,40,41}$. According to them, in contrast to healthy individuals, CLBP participants may avoid activating muscles that are required to create specific body movements and forces. In fact, it indicates a constraint during the recovery period after voluntary arm movement to prevent falling. This results in a smaller COP excursion, decreasing the driving force to return to equilibrium ${ }^{42}$. This may explain why differences were not observed between both groups. Meanwhile, the time to reach the peak of COP displacement was significantly shorter in the control elderly group, indicating that the healthy elderly participants were able to recover their balance faster than those with CLPB. 
This outcome is consistent with findings previously reported in adults with and without CLBP ${ }^{13,40}$, as it is well known that increased time for postural stabilization is associated with poor postural control ${ }^{13,32,40}$. Therefore, our COP results demonstrate a reduction in the quality of postural recovery in elderly individuals with CLBP.

Some theoretical models support our findings related to muscles and COP. First, patients with CLBP have an altered proprioception in their lumbar-pelvic region, which resulted in difficulties in the calculations of the initial or final positions of body segments or in reproducing a previously set position ${ }^{43}$. Owing to the lack of feedback from their lumbar spine (i.e., the spine position was uncertain), CLBP patients presented ineffective control of their COM position to use the hip strategy in postural control (i.e., when lumbo-pelvic movement was involved). The hip strategy is complex and requires the interpretation of angle changes at the hip and spine to calculate the COM position ${ }^{44}$. This may explain why the CLBP elderly individuals performed worse when using the hip strategy ${ }^{36}$.

Altered postural muscle control was described in CLPB participants as a mechanism to minimize trunk motion and maintain protective stiffness to avoid pain or successive injuries ${ }^{43,45,46}$. Moreover, the fear of falling, typically present in elderly people, may alter muscle control in a protective manner ${ }^{32}$. An altered timing of muscle activation and increased co-contraction in the axial muscles during body perturbation has been reported in individuals with CLBP $25,34,37,45$. This increases spinal stiffness and reduces spinal movement ${ }^{47-49}$.

Finally, when performing rapid voluntary arm movements, the CLBP elderly participants presented impaired APAs when compared with the control group. Delayed lower limb proximal and SOL muscles and COP onset highlighted this condition. In addition, their inability to achieve timely postural recovery (i.e., higher $\left.\mathrm{COP}_{\text {timetopeak }}\right)$ reflected delayed postural control during the compensatory phase. Hence, elderly individuals with CLBP might encounter a higher risk of falling under situations that require rapid recovery. Clinical assessment for this population should account for postural stability during rehabilitation programs.

\section{Data availability}

Data available as "Supplementary files".

Received: 5 June 2020; Accepted: 9 February 2021

Published online: 26 February 2021

\section{References}

1. Airaksinen, O. et al. Chapter 4: European guidelines for the management of chronic nonspecific low back pain. Eur. Spine J. 15 (2006).

2. de Souza, I. M. B., Sakaguchi, T. F., Yuan, S. L. K., Matsutani, L. A., do Espírito-Santo, A. S., Pereira, C. A. B. \& Marques, A. P. Prevalence of low back pain in the elderly population: A systematic review. Clinics 74, e789. https://doi.org/10.6061/clinics/2019/ e789 (2019)

3. Edmond, S. L. \& Felson, D. T. Function and back symptoms in older adults. J. Am. Geriatr. Soc. 51, 1702-1709 (2003).

4. Cooper, J. K. \& Kohlmann, T. Factors associated with health status of older Americans. Age Ageing 30, 495-501 (2001).

5. Hoy, D. et al. The global burden of low back pain: Estimates from the Global Burden of Disease 2010 study. Ann. Rheum. Dis. 73, 968-974 (2014).

6. Knox, M. F., Chipchase, L. S., Schabrun, S. M., Romero, R. J. \& Marshall, P. W. M. Anticipatory and compensatory postural adjustments in people with low back pain: A systematic review and meta-analysis. Spine J. https://doi.org/10.1016/j.spinee.2018.06.008 (2018).

7. Kanekar, N. \& Aruin, A. S. The effect of aging on anticipatory postural control. Exp. Brain Res. https://doi.org/10.1007/s00221014-3822-3 (2014).

8. Bleuse, S. et al. Effect of age on anticipatory postural adjustments in unilateral arm movement. Gait Posture 24, 203-210 (2006).

9. Santos, M. J., Kanekar, N. \& Aruin, A. S. The role of anticipatory postural adjustments in compensatory control of posture: 1 . Electromyographic analysis. J. Electromyogr. Kinesiol. 20, 388-397 (2010)

10. Santos, M. J., Kanekar, N. \& Aruin, A. S. The role of anticipatory postural adjustments in compensatory control of posture: 2. Biomechanical analysis. J. Electromyogr. Kinesiol. 20, 398-405 (2010).

11. Teyssedre, C., Lino, F., Zattara, M. \& Bouisset, S. Anticipatory EMG patterns associated with preferred and non-preferred arm pointing movements. Exp. Brain Res. 134, 435-440 (2000).

12. Massion, J. Movement, posture and equilibrium: Interaction and coordination. Prog. Neurobiol. 38, 35-56 (1992)

13. Mok, N. W., Brauer, S. G. \& Hodges, P. W. Postural recovery following voluntary arm movement is impaired in people with chronic low back pain. Gait Posture 34, 97-102 (2011).

14. Lee, Y. J., Chen, B. \& Aruin, A. S. Older adults utilize less efficient postural control when performing pushing task. J. Electromyogr. Kinesiol. 25, 966-972 (2015).

15. Skelton, D. A. \& Beyer, N. Exercise and injury prevention in older people. Scand. J. Med. Sci. Sport. 13, 77-85 (2003).

16. Kubicki, A., Fautrelle, L., Bourrelier, J., Rouaud, O. \& Mourey, F. The early indicators of functional decrease in mild cognitive impairment. Front. Aging Neurosci. 8, 1-11 (2016).

17. Woollacott, M. H. \& Manchester, D. L. Anticipatory postural adjustments in older adults: Are changes in response characteristics due to changes in strategy?. J. Gerontol. 48, M64-70 (1993).

18. Silfies, S. P., Mehta, R., Smith, S. S. \& Karduna, A. R. Differences in feedforward trunk muscle activity in subgroups of patients with mechanical low back pain. Arch. Phys. Med. Rehabil. https://doi.org/10.1016/j.apmr.2008.10.033 (2009).

19. Mehta, R., Cannella, M., Smith, S. \& Silfies, S. Altered trunk motor planning in patients with nonspecific low back pain. J. Mot. Behav. 42, 135-144 (2010).

20. Akbari, M., Sarrafzadeh, J., Maroufi, N. \& Haghani, H. Changes in postural and trunk muscles responses in patients with chronic nonspecific low back pain during sudden upper limb loading. Med. J. Islam. Repub. Iran 29, 833-838 (2015).

21. Mok, N. W., Brauer, S. G. \& Hodges, P. W. Changes in lumbar movement in people with low back pain are related to compromised balance. Spine (Phila. Pa. 1976). 36, 45-52 (2011).

22. Massé-Alarie, H., Beaulieu, L. D., Preuss, R. \& Schneider, C. Task-specificity of bilateral anticipatory activation of the deep abdominal muscles in healthy and chronic low back pain populations. Gait Posture 41, 440-447 (2015).

23. Massé-Alarie, H., Beaulieu, L. D., Preuss, R. \& Schneider, C. The side of chronic low back pain matters: Evidence from the primary motor cortex excitability and the postural adjustments of multifidi muscles. Exp. Brain Res. 235, 647-659 (2017).

24. Hedayati, R. et al. The study of the variability of anticipatory postural adjustments in patients with recurrent non-specific low back pain. J. Back Musculoskelet. Rehabil. 27, 33-40 (2014). 
25. Sadeghi, M., Talebian, S., Olyaei, G. R. \& Attarbashi Moghadam, B. Preparatory brain activity and anticipatory postural adjustments accompanied by externally cued weighted-rapid arm rise task in non-specific chronic low back pain patients and healthy subjects. Springerplus 5(1), 674. https://doi.org/10.1186/s40064-016-2342-y (2016).

26. Roach, K. E. et al. The sensitivity and specificity of pain response to activity and position in categorizing patients with low back pain. Phys. Ther. 77, 730-738 (1997).

27. Ferreira-Valente, M. A., Pais-Ribeiro, J. L. \& Jensen, M. P. Validity of four pain intensity rating scales. Pain 152, 2399-2404 (2011).

28. Fairbank, J. C. T. \& Pynsent, P. B. The oswestry disability index. Spine (Phila. Pa. 1976). https://doi.org/10.1097/00007632-20001 1150-00017 (2000).

29. Hermens, H. J., Freriks, B., Disselhorst-Klug, C. \& Rau, G. Development of recommendations for SEMG sensors and sensor placement procedures. J. Electromyogr. Kinesiol. 10, 361-374 (2000).

30. Papaxanthis, C., Pozzo, T. \& Schieppati, M. Trajectories of arm pointing movements on the sagittal plane vary with both direction and speed. Exp. Brain Res. 148, 498-503 (2003).

31. Winter, D. A. Human balance and posture control during standing and walking. Gait Posture https://doi.org/10.1016/0966-6362(96) 82849-9 (1995).

32. Brauer, S. G., Woollacott, M. \& Shumway-Cook, A. The interacting effects of cognitive demand and recovery of postural stability in balance-impaired elderly persons. J. Gerontol. Ser. A Biol. Sci. Med. Sci. 56, M489-M496 (2001).

33. Matsui, K., Tachibana, T., Nobuhara, K. \& Uchiyama, Y. Translational movement within the glenohumeral joint at different rotation velocities as seen by cine MRI. J. Exp. Orthop. https://doi.org/10.1186/s40634-018-0124-x (2018).

34. Jacobs, J. V., Henry, S. M. \& Nagle, K. J. People with chronic low back pain exhibit decreased variability in the timing of their anticipatory postural adjustments. Behav. Neurosci. 123, 455-458 (2009).

35. Hemmati, L., Piroozi, S. \& Rojhani-Shirazi, Z. Effect of dual tasking on anticipatory and compensatory postural adjustments in response to external perturbations in individuals with nonspecific chronic low back pain: Electromyographic analysis. J. Back Musculoskelet. Rehabil. 31, 489-497 (2018).

36. Mok, N. W., Brauer, S. G. \& Hodges, P. W. Hip strategy for balance control in quiet standing is reduced in people with low back pain. Spine (Phila. Pa. 1976). 29, E107-E112 (2004).

37. Radebold, A., Cholewicki, J., Panjabi, M. M. \& Patel, T. C. Muscle response pattern to sudden trunk loading in healthy individuals and in patients with chronic low back pain. Spine (Phila. Pa. 1976). 25, 947-954 (2000).

38. Kennedy, A., Bugnariu, N., Guevel, A. \& Sveistrup, H. Adaptation of the feedforward postural response to repeated continuous postural perturbations. Neurosci. Med. https://doi.org/10.4236/nm.2013.41007 (2013).

39. Chiovetto, E., Berret, B. \& Pozzo, T. Tri-dimensional and triphasic muscle organization of whole-body pointing movements. Neuroscience 170, 1223-1238 (2010).

40. Etemadi, Y., Salavati, M., Arab, A. M. \& Ghanavati, T. Balance recovery reactions in individuals with recurrent nonspecific low back pain: Effect of attention. Gait Posture 44, 123-127 (2016).

41. Henry, S. M., Hitt, J. R., Jones, S. L. \& Bunn, J. Y. Decreased limits of stability in response to postural perturbations in subjects with low back pain. Clin. Biomech. 21, 881-892 (2006).

42. Corriveau, H., Heébert, R., Prince, F. \& Rache, M. Postural control in the elderly: An analysis of test-retest and interrater reliability of the COP-COM variable. Arch. Phys. Med. Rehabil. 82, 80-85 (2001).

43. Gill, K. P. \& Callaghan, M. J. The measurement of lumbar proprioception in individuals with and without low back pain. Spine 23, 371-377 (1998).

44. Nashner, L. M., Shupert, C. L. \& Horak, F. B. Head-trunk movement coordination in the standing posture. Prog. Brain Res. 76, 243-251 (1988).

45. Van Dieën, J. H., Cholewicki, J. \& Radebold, A. Trunk muscle recruitment patterns in patients with low back pain enhance the stability of the lumbar spine. Spine (Phila. Pa. 1976). 28, 834-841 (2003).

46. Brumagne, S., Cordo, P., Lysens, R., Verschueren, S. \& Swinnen, S. The role of paraspinal muscle spindles in lumbosacral position sense in individuals with and without low back pain. Spine (Phila. Pa. 1976). 25, 989-994 (2000).

47. Cholewicki, J., Panjabi, M. M. \& Khachatryan, A. Stabilizing function of trunk flexor-extensor muscles around a neutral spine posture. Spine 22, 2207-2212 (1997).

48. Gardner-Morse, M. G. \& Stokes, I. A. F. Trunk stiffness increases with steady-state effort. J. Biomech. 34, 457-463 (2001).

49. Mok, N. W., Brauer, S. G. \& Hodges, P. W. Failure to use movement in postural strategies leads to increased spinal displacement in low back pain. Spine (Phila. Pa. 1976). 32(19), E537-E43. https://doi.org/10.1097/BRS.0b013e31814541a2 (2007).

\section{Acknowledgements}

We thank Bruno Giovanni Afonso da Silva and Bruna Callegari for the development of Figure 1.

\section{Author contributions}

Conceived and designed the experiments: B.C., D.R.G. Performed the experiments: B.C., D.R.G., G.C.S.A., C.F.O.S., T.S.N. Analyzed the data: B.C., D.R.G., G.S.S., A.A.C.S. Contributed with materials and analysis tools: B.C., G.S.S., E.S.Y. Wrote the paper: D.R.G., A.K., B.C., E.S.Y. Approved the final version to be submitted: D.R.G., B.C., G.C.S.A., C.F.O.S., T.S.N., A.A.C.S., A.R.K., G.S.S., E.S.Y.

\section{Funding}

This research was supported by the following Grants: Pará Amazon Research Support Foundation (FAPESPA) Grant number \#180/2012; and Coordination for the Improvement of Higher Education Personnel (CAPES)/ COFECUB research Grant number \#819-14. The funders had no role in study design, data collection and analysis, decision to publish, or preparation of the manuscript. Conselho Nacional de Desenvolvimento Científico e Tecnológico (CNPQ).

\section{Competing interests}

The authors declare no competing interests.

\section{Additional information}

Supplementary Information The online version contains supplementary material available at https://doi.org/ 10.1038/s41598-021-83837-2.

Correspondence and requests for materials should be addressed to B.C.

Reprints and permissions information is available at www.nature.com/reprints. 
Publisher's note Springer Nature remains neutral with regard to jurisdictional claims in published maps and institutional affiliations.

(c) (i) Open Access This article is licensed under a Creative Commons Attribution 4.0 International License, which permits use, sharing, adaptation, distribution and reproduction in any medium or format, as long as you give appropriate credit to the original author(s) and the source, provide a link to the Creative Commons licence, and indicate if changes were made. The images or other third party material in this article are included in the article's Creative Commons licence, unless indicated otherwise in a credit line to the material. If material is not included in the article's Creative Commons licence and your intended use is not permitted by statutory regulation or exceeds the permitted use, you will need to obtain permission directly from the copyright holder. To view a copy of this licence, visit http://creativecommons.org/licenses/by/4.0/.

(C) The Author(s) 2021, corrected publication 2021 